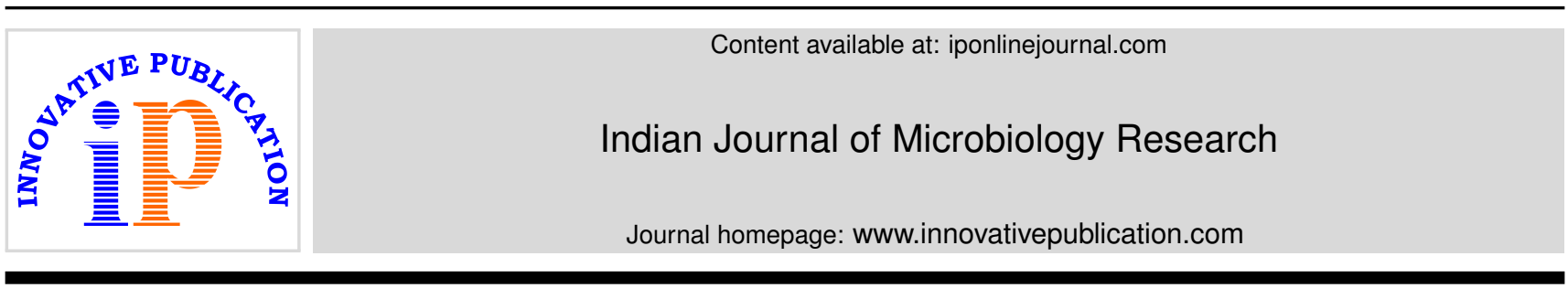

Original Research Article

\title{
A study to analyze the differences between GeneXpert MTB/RIF assay and LED fluorescent microscopy for the diagnosis of pulmonary tuberculosis in a tertiary care centre
}

\author{
Thomas S Kuruvilla ${ }^{1} *$, Pampi Majumder ${ }^{2}$ \\ ${ }^{1}$ Dept. of Microbiology, Father Muller Medical College, Mangalore, Karnataka, India \\ ${ }^{2}$ Suraksha Diagnostic Pvt. Ltd, Kolkata, West Bengal, India
}

\section{A R T I C L E I N F O}

\section{Article history:}

Received 20-01-2020

Accepted 20-02-2020

Available online 08-04-2020

Keywords:

LED fluorescent microscopy

GeneXpert

Rifampicin resistance

\begin{abstract}
A B S T R A C T
Introduction: Mycobacterium tuberculosis cultures although being the gold standard for diagnosing tuberculosis has its limitations as it is time consuming. Thus newer methods using light-emitting diode fluorescent microscopy (LED-FM) and molecular methods like the GeneXpert MTB/RIF assay are being adopted as a mainstay for diagnosing tuberculosis around the globe.

Materials and Methods: This observational descriptive study was carried out for a period of 21 months. Sputum samples from 110 adult subjects with a clinical suspicion of pulmonary tuberculosis (PTB) were stained for LED-FM microscopy and also inoculated into the GeneXpert assay. The positive smears were graded as per the Revised National Tuberculosis Control Programme (RNTCP) guidelines and positive GeneXpert assay results along with Rifampicin resistance were noted and analysed.

Results: Out of the 110 samples, (77.2\%) were males and (22.7\%) females. Of the 110 samples, 75 (68.1\%) were positive for Mycobacterium tuberculosis by the GeneXpert assay. In contrast with LED-FM, all 25 smear positive samples were also GeneXpert positive. Additionally, GeneXpert detected 10 smear negative cases. $10(9 \%)$ positive GeneXpert isolates showed resistance to Rifampicin. The overall sensitivity of GeneXpert was greater but its specificity was comparable with LED-FM.

Conclusion: The Xpert and LED-FM tests were comparable in specificity, but the GeneXpert assay out did the LED-FM as it was a more sensitive and rapid test for the diagnosis of tuberculosis and additionally detection of Rifampicin resistance. Our study fully agrees that the GeneXpert assay is a step ahead in the early diagnosis of tuberculosis even for smear negative cases of PTB. This assay is also a relatively simple and routine test that staff can perform with minimal training.
\end{abstract}

(C) 2020 Published by Innovative Publication. This is an open access article under the CC BY-NC-ND license (https://creativecommons.org/licenses/by/4.0/)

\section{Introduction}

Mycobacterium tuberculosis remains one of the most significant causes of death from an infectious agent. ${ }^{1}$ Tuberculosis (TB) is a public health hazard in India and in many other countries. ${ }^{2}$ Culture techniques are still considered the gold standard to diagnose Mycobacterium tuberculosis. ${ }^{1}$ The delay in growing Mycobacteria invariably prolongs the clinical approach to the patient and advanced laboratory facilities may not be affordable for

\footnotetext{
* Corresponding author.

E-mail address: thomssk@yahoo.com (T. S. Kuruvilla).
}

many in most of the rural health centres. ${ }^{1}$ The GeneXpert MTB/RIF assay is a relatively new fully automated test based on the principle of nucleic acid amplification. Apart from identifying Mycobacterium tuberculosis it can also detect Rifampicin resistance. ${ }^{1}$ Auramine-O stained sputum smear examined by LED microscopy was developed mainly to give resource-limited countries access to the benefits of fluorescence microscopy. ${ }^{3}$ They have better sensitivity and efficiency when compared to conventional fluorescent microscopy. ${ }^{3}$ Our study analyzes the clinical utility of the GeneXpert assay with LED-FM for the diagnosis of pulmonary tuberculosis. 
The GeneXpert assay is a novel fully automated molecular test that can analyse the sensitivity of five genome copies of purified DNA and $131 \mathrm{cfu} / \mathrm{ml}$ of M. tuberculosis. ${ }^{4}$ It was introduced by Cepheid, Inc., the Foundation for Innovative New Diagnostics (FIND), University of Medicine and Dentistry of New Jersey ( UMDNJ ), and the National Institutes of Health (NIH). The World Health Organization (WHO) has found its clinical utility for the diagnosis of pulmonary tuberculosis even among HIV infected patients from developing countries. ${ }^{4}$ The GeneXpert MTB/RIF assay is more precise in smear-positive specimens and its sensitivity to diagnosing pulmonary tuberculosis in adults was more than that in children $(90.8 \%$ versus $74.3 \%)$ in a study by Chang et al. ${ }^{5}$

GeneXpert MTB/RIF increased TB case detection, especially in patients with advanced immunosuppression in a study by Balcha et al. ${ }^{6} \mathrm{He}$ said that an algorithm based on the use of a single morning sputum sample for individuals with negative sputum smear microscopy could be considered for intensified case finding in patients eligible for antiretroviral therapy. ${ }^{6 T}$ he GeneXpert MTB/RIF assay and nested PCR technique have a more or less similar sensitivity and specificity to the for the routine diagnosis of Mycobacterial culture-positive TB. ${ }^{7}$ The molecular method also allows quicker clinical judgements and faster initiation of antitubercular treatment. ${ }^{7}$

The aim of our study was to analyze the differences between GeneXpert MTB/RIF assay and LED-FM for the diagnosis of pulmonary tuberculosis and identify drug resistance Mycobacterium isolates with reference to Rifampicin.

\section{Materials and Methods}

This observational descriptive prospective study was carried out in the microbiology laboratory for a period of 21 months from Mar 2017 to Dec 2018 after ethical clearance. A well coughed up sputum samples from suspected cases of pulmonary tuberculosis (PTB) or samples obtained by invasive methods (Bronchoalveolar lavage i.e. BAL, endotracheal suction tips \& gastric aspirates) were processed. 110 adults suspected to have PTB and those that were on anti-tubercular treatment (ATT) for not more than two weeks (in and out patients) were included in the study and all adults suspected to have extrapulmonary tuberculosis and those cases from who have had ATT for more than 2 weeks were excluded from the study. ${ }^{8}$ After collection of all demographic and clinical details of the patients in our study, their respiratory samples were subjected to staining and interpretation as per the protocol laid down by the RNTCP guidelines for LED-FM and as per standard operating procedures outlined in the working manual of the GeneXpert TB assay. ${ }^{9}$

\subsection{Auramine-rhodamine staining process for Mycobacterium tuberculosis}

The fixed smear was placed on a staining rack and the slide was stained with auramine-rhodamine foraround 15 minutes without letting the surface to dry up. The stain was washed off with distilled water. A fluorescent decolorizer (i.e acidalcohol) was added for 2-3 minutes and the slide was again washed with distilled water. Potassium permanganate (counter stain) was then added for 3-4 minutes. The slide was finally washed with distilled water and air dried and the observed under LED fluorescent microscopy. Grading of the bacilli was done as per the latest RNTCP LED-FM guidelines ${ }^{9}$ as depicted in (Table 1 )

\subsection{GeneXpert assay}

The respiratory sample was mixed with sample reagent that is provided with the assay (containing $\mathrm{NaOH}$ and isopropyl alcohol) at a 2:1 ratio to clinical specimen to kill the Mycobacteria and liquefy the samples with the reagent. Fluids like (BAL) were processed directly by the addition of a 2:1 volume of SR buffer. The sample-SR mixture was shaken vigorously and incubated for 10 minutes before being shaken again and kept at room temperature for another 10 minutes. $2 \mathrm{ml}$ of the digested material was transferred to the cartridge. The cartridge was subsequently loaded in the GeneXpert instrument where all subsequent steps occurred automatically and results read in real time. In case the results were reported as invalid, error or no result, the sample was reprocessed and rerun, if sufficient material was available. The tuberculosis bacilli once detected will be tested for Rifampicin resistance in the same assay. ${ }^{10}$ The selected data was analysed by frequency, percentage and the Chi square test.

\section{Results}

In comparison of GeneXpert with LED-FM, all 25 smear positive samples were GeneXpert positive. In addition, GeneXpert detected 10 of the smear negative cases. Of the 110 specimens that yielded a valid GeneXpert MTB/RIF result, 10 (9\%) positive GeneXpert isolates showed resistance to Rifampicin of the sputum isolates.

The various statistical comparative values are shown in (Table 4). There is an appreciable increase in positive results of GeneXpert MTB/RIF with LED fluorescent microscopy when performing both the tests on the same sputum sample.

\section{Discussion}

Tuberculosis is one of the oldest diseases in the world associated with high morbidity and mortality. ${ }^{11}$ India accounts for one-fourth of the global TB burden with an estimated 2.79 million incident cases. ${ }^{11}$ A reason for the significant prevalence of this disease is the difficulty in 
Table 1: RNTCP LED FM smear grading protocol

\begin{tabular}{llll}
\hline S.No & Min Number of Fields To & LED-FM 1 Length = 40 Fields & Result \\
1 & Examine & Zero AFB / 1 Length & Negative \\
2 & 40 & $1-19$ AFB / 1 Length & Scanty \\
3 & 40 & $20-199$ AFB / 1 Length & $1+$ \\
4 & 40 & $5-50$ AFB / 1 Field on average & $2+$ \\
5 & 20 & $>50$ AFB / 1 Field on average & $3+$ \\
\hline
\end{tabular}

Table 2: Age \& Sex wise distribution of the cases

\begin{tabular}{llll}
\hline S.No & Age $($ yrs $)$ & Male & Female \\
$\mathbf{1}$ & $20-30$ & 08 & 02 \\
$\mathbf{2}$ & $31-40$ & 12 & 05 \\
$\mathbf{3}$ & $41-50$ & 13 & 06 \\
$\mathbf{4}$ & $51-60$ & 24 & 06 \\
$\mathbf{5}$ & $>60$ & 28 & 07 \\
Total & & $\mathbf{8 5}$ & $\mathbf{2 5}$
\end{tabular}

Table 3: Sample wise comparison ofGeneXpert \& LED Microscopy

\begin{tabular}{lllll}
\hline S.No & Sample & $\begin{array}{l}\text { Positive Gene XPERT } \\
\text { ASSAY }\end{array}$ & Positive LED-FM & Negative LED-FM \\
$\mathbf{1}$ & Sputum & 50 & 17 & 5 \\
$\mathbf{2}$ & BAL & 10 & 2 & 2 \\
$\mathbf{3}$ & ET Tip & 7 & 2 & 1 \\
$\mathbf{4}$ & Gastric aspirates & 8 & 4 & 2 \\
Total & & $\mathbf{7 5}$ & $\mathbf{2 5}$ & $\mathbf{1 0}$ \\
\hline
\end{tabular}

Table 4: The Sensitivity, Specificity, Positive Predictive value (PPV) \& Negative predictive value (NPV) of both tests.

\begin{tabular}{lllll}
\hline Methods & Sensitivity (\%) & Specificity (\%) & PPV(\%) & NPV(\%) \\
Xpert Assay & 96.1 & 98 & 94.1 & 99.1 \\
LED- FM & 46.2 & 99.1 & 95.3 & 87.2 \\
\hline
\end{tabular}

diagnosis. Traditionally, Mycobacterial culture is the gold standard for the diagnosis of tuberculosis. ${ }^{12}$ This approach is relatively labour intensive and takes about 2 months before results are available. ${ }^{13}$

The diagnostic performance of GeneXpert assay was very evident in our study both in specificity (98\%) and sensitivity (96.1\%). Our results were comparable with similar studies by Danish et al $^{13}$ where their GeneXpert test was quite sensitive when compared to culture for not only in smear positive and smear negative but also in extrapulmonary tuberculosis. He also found the GeneXpert assay to be user friendly and can be performed with minimal training. ${ }^{13}$ A better detection rate of GeneXpert was reported in a study when compared to smear microscopy and culture by Bates et al. ${ }^{10,14}$ Our results revealed that the detection rate (specificity and sensitivity) of the tested samples for GeneXpert MTB/RIF assay was almost two to three fold to that of LED-FM microscopy.

In a study by Gerardo et al, they found that smear positivity by LED microscopy to be $63.9 \%$ and $74.7 \%$ by GeneXpert. ${ }^{4}$ Another study done to validate GeneXpert was by Chang $\mathrm{K}$ et $\mathrm{al}^{5}$ revealed that the summary estimate was
90.4\% for sensitivity, $98.4 \%$ for specificity in pulmonary tuberculosis. ${ }^{10}$ The sensitivity and specificity of detecting RIF-resistance was $94.1 \%, 97.0 \%$ respectively. ${ }^{5}$

The current conventional techniques, i.e. LED-FM and culture system not only need a Biosafety Level III (BSLIII) and trained personnel but also cultures may take several weeks to yield results. The GeneXpert MTB/RIF being a proven technology does not require special user's skills and routine technologists with minimal training can perform the test. ${ }^{1,4}$ An ultra short turnaround time and simultaneous detection of Rifampicin (RIF) resistance within 3 hours gives the GeneXpert assay an added advantage. ${ }^{1}$ A conventional laboratory technique such as LED-FM smear microscopy are cost effective but less sensitive as a large bacillary load $\left(10^{5} / \mathrm{mL}\right)$ will be required for smear positivity. ${ }^{1,4}$

In a study done in South Africa with HIV-infected patients, the GeneXpert MTB/RIF showed an increase in case detection for tuberculosis by $45 \%$ when compared to fluorescent microscopy. Here there were differences in the populations involved in the studies and also the way 
sputum samples were collected. However in this study, two sputum samples were collected in a single visit to outpatient clinics before the initiation of antiretroviral treatment and regardless of symptoms. ${ }^{4,15}$

Our study has some limitations. Apart from cost factor considerations of molecular method, we have not performed Mycobacterial culture to rule out false positive results. However, both these tests have shown specificities above $95 \%$ in studies by Vadwai, Parsons and Tortoli. et al. ${ }^{4,16-18}$ Thus there could be a possibility of bias due to false positive results but it can be considered insignificant. The latest technology of the GeneXpert MTB/RIF is the GeneXpert Ultra. This is a step ahead in diagnostics of pulmonary tuberculosis with a bacilli minimum requirement of 15.6 $\mathrm{cfu} / \mathrm{ml}$ of sample. The transition to this new cartridge system will slowly replace the older format increasing the chances of diagnosis of tuberculosis particularly when dealing with smear negative cases and multi drug resistant Mycobacteria. ${ }^{19}$

\section{Conclusion}

The GeneXpert MTB/RIF assay beyond doubt has raised the standards of rapid diagnosis of tuberculosis. In a setting where this assay is not affordable, the LED fluorescent microscopy can still be used with acceptable results particularly if more than one sputum sample can be collected.

\section{Source of Funding}

None.

\section{Conflict of Interest}

None.

\section{References}

1. Zeka AN, Tasbakan S, Cavusoglu C. Evaluation of the GeneXpert MTB/RIF Assay for Rapid Diagnosis of Tuberculosis and Detection of Rifampin Resistance in Pulmonary and Extrapulmonary Specimens. J Clin Microbiol. 2011;49(12):4138-41.

2. Akther S, Fernandes H, Goyal G. Comparison of auramine$\mathrm{O}$ stain with Zeihl-Neelson stain in suspected cases of tubercular lymphadenitis. Int J Recent Trends Sci Tech. 2015;15(3):524-5.

3. Dezemon Z, Muvunyi CM, Jacob O. Staining techniques for detection of acid fast bacilli: what hope does fluorescein-diacetate (FDA) vitality staining technique represent for the monitoring of tuberculosis treatment in resource limited settings. Trends Bact. 2014;1(1):1-1.

4. Alvarez-Uria G, Azcona JM, Midde M, Naik PK, Reddy S, Reddy R. Rapid Diagnosis of Pulmonary and Extrapulmonary Tuberculosis in HIV-Infected Patients. Comparison of LED Fluorescent Microscopy and the GeneXpert MTB/RIF Assay in a District Hospital in India. Tuberculosis Res Treat. 2012;2012:1-4.

5. Chang K, Lu W, Wang J, Zhang K, Jia S, et al. Rapid and effective diagnosis of tuberculosis and rifampicin resistance with Xpert MTB/RIF assay: A meta-analysis. J of Inf. 2012;64(6):580-8.
6. Balcha TT, Sturegård $\mathrm{E}$, Winqvist $\mathrm{N}$, Skogmar S, Reepalu A, et al. Intensified Tuberculosis Case-Finding in HIV-Positive Adults Managed at Ethiopian Health Centers: Diagnostic Yield of Xpert MTB/RIF Compared with Smear Microscopy and Liquid Culture. PLoS ONE. 2014;9(1):e85478.

7. Kim $\mathrm{CH}$, Woo H, Hyun $\mathrm{IG}$, Kim C, Choi JH, et al. A comparison between the efficiency of the Xpert MTB/RIF assay and nested PCR in identifying Mycobacterium tuberculosis during routine clinical practice. J Thor Med. 2014;6(6):625-31.

8. Surendra K, Sharma M, Kohli R, Yadav JN, Chaubey D, Bhasin V. PLOS ONE;doi:10.1371/journal.pone.014101]

9. Ahmed S, Shukla I, Fatima N, Varshney SK, Shameem M, Tayyaba U. Light-Emitting Diode-Fluorescent Microscopy: Determining its Sensitivity and Specificity in Diagnosis of Pulmonary Tuberculosis in a High-Burden Tuberculosis Region and Resource-Limited Country like India. CHRISMED J Health Res. 2019;6(1):44-51.

10. Khana AS, Ali S, Khan MT, Ahmed S, Khattak Y, et al. Comparison of GeneXpert MTB/RIF assay and LED-FM microscopy for the diagnosis of extra pulmonary tuberculosis in Khyber Pakhtunkhwa, Pakistan. Braz J of Microbiol . 2018;49:909-13.

11. Ramana BV, Srikar A, Prakash P, Chaudhury A. Diagnostic accuracy of XpertMTB/RIF assay in detection of Pulmonary tuberculosis and Rifampicin resistance in comparison with conventional methods. Int J Sci Res. 2019;8(10):37-9.

12. Iram S, Zeenat A, Hussain S, Yusuf NW, Aslam M. Rapid diagnosis of tuberculosis using Xpert MTB/RIF assay - Report from a developing country. Pak J Med Sci. 2015;31(1):105-10.

13. Zahoor D, Farhana A, Kanth F, Bha M. Evaluation of smear microscopy and geneXpert for the rapid diagnosis of pulmonary and extrapulmonary tuberculosis in a tertiary care hospital in North India: a descriptive prospective study. Int J Res Med Sci. 2018;6(5):1756-60.

14. Bates M, O’Grady J, Maeurer M, Tembo J, Chilukutu L, et al. Assessment of the Xpert MTB/RIF assay for diagnosis of tuberculosis with gastric lavage aspirates in children in sub-Saharan Africa: a prospective descriptive study. Lancet Infect Dis. 2013;13(1):36-42.

15. Lawn SD, Brooks SV, Kranzer K, Nicol MP, Whitelaw A, et al. Screening for HIV-Associated Tuberculosis and Rifampicin Resistance before Antiretroviral Therapy Using the Xpert MTB/RIF Assay: A Prospective Study. PLoS Med. 2011;8(7). [1001067.

16. Vadwai V, Boehme C, Nabeta P, Shetty A, Alland D, et al. Xpert MTB/RIF: a New Pillar in Diagnosis of Extrapulmonary Tuberculosis? J Clin Microbiol. 2011;49(7):2540-5.

17. Parsons LM, Somoskovi A, Gutierrez C, Lee E, Paramasivan CN, et al Laboratory Diagnosis of Tuberculosis in Resource-Poor Countries: Challenges and Opportunities. Clin Microbiol Rev. 2011;24(2):31450 .

18. Tortoli E, Russo C, Piersimoni C, Mazzola E, Monte PD, et al. Clinical validation of Xpert MTB/RIF for the diagnosis of extrapulmonary tuberculosis. Euro Resp J. 2012;40(2):442-7.

19. Opota O, Mazza-Stalder J, Greub G, Jaton K. The rapid molecular test Xpert MTB/RIF ultra: towards improved tuberculosis diagnosis and rifampicin resistance detection. Clin Microbiol Inf. 2019;25(11):1370-6.

\section{Author biography}

Thomas S Kuruvilla Associate Professor

Pampi Majumder Consultant Microbiologist

Cite this article: Kuruvilla TS, Majumder P. A study to analyze the differences between GeneXpert MTB/RIF assay and LED fluorescent microscopy for the diagnosis of pulmonary tuberculosis in a tertiary care centre. Indian J Microbiol Res 2020;7(1):39-42. 